\title{
Coil Protection Using Small Helical Coils for Wide-Neck Intracranial Aneurysms: A Novel Approach
}

\author{
Y.D. Cho, J.Y. Lee, J.H. Seo, S.J. Lee, H.-S. Kang, J.E. Kim, O.-K. Kwon, Y.J. Son, and M.H. Han
}

\begin{abstract}
BACKGROUND AND PURPOSE: A number of remodeling or protective techniques available to treat wide-neck intracranial aneurysms are increasingly being used, provided that the shape/type of aneurysm, vessel diameter, and inherent course of the vessel are conducive to their use. The purpose of this study was to describe a novel method using coil protection for treatment of wide-neck aneurysms.
\end{abstract}

MATERIALS AND METHODS: This technique involves sequential maneuvers to the aneurysm and affected branch artery. A microcatheter is first introduced into the aneurysmal sac, and another microcatheter is introduced into the entrance of the branch artery, followed by partial deployment of a small helical coil into the branch artery. A framing coil is then placed within the aneurysmal sac, under the protection of the helical coil. After completion of the first coil insertion, the helical coil should be retrieved to confirm the stability of the framing coil. The helical coil can also serve as a filler.

RESULTS: This technique was successfully applied to 12 intracranial saccular aneurysms of the MCA bifurcation (5 patients); anterior communicating artery (3 patients); and A1 and M1 segments, distal ACA, and basilar tip (1 patient each). Selective endovascular treatment was successfully performed and resulted in excellent outcomes in all patients. There were no complications directly related to coil protection.

CONCLUSIONS: Our small study suggests that coil protection can be a safe alternative to traditional remodeling or protective techniques when those techniques have failed or are not possible due to vascular geometry. It is particularly suited for the treatment of wide-neck aneurysms arising from small and acutely angulated branching vessels.

ABBREVIATION: ACA = anterior cerebral artery

$\mathrm{S}$ hallow aneurysms with wide necks pose a technical challenge for endovascular treatment. ${ }^{1-3}$ Newer techniques incorporating balloon or stent remodeling, multiple microcatheters, and microcatheter protection have enabled the treatment by coil embolization of aneurysms with complex configurations. ${ }^{1-8} \mathrm{Al}-$ though such options have significantly improved the prospect of endovascular therapy in this setting, some lesions are not amenable to treatment by virtue of aneurysmal configuration and the

Received January 17, 2012; accepted after revision March 16.

From the Departments of Radiology (Y.D.C., J.Y.L., J.H.S., S.J.L., M.H.H.) and Neurosurgery (H.-S.K., J.E.K., M.H.H.), Seoul National University Hospital, Department of Neurosurgery (O.-K.K.), Seoul National University Bundang Hospital, and Department of Neurosurgery (Y.J.S.), Seoul National University Boramae Medical Center, Seoul National University College of Medicine, Seoul, Korea.

This study was supported by a grant from the Korea Healthcare Technology R\&D Project, Ministry for Health, Welfare and Family Affairs, Republic of Korea (A111101) Please address correspondence to Moon Hee Han, MD, PhD, Departments of Radiology and Neurosurgery, Seoul National University College of Medicine, 28 Yongon-dong, Jongno-gu, Seoul, Korea; e-mail: hanmh@snuh.org

-- Indicates open access to non-subscribers at www.ajnr.org

http://dx.doi.org/10.3174/ajnr.A3157 course or caliber of the branch artery. Therefore, using a small protective helical coil, we have developed a new approach for wide-neck aneurysms at entries of small and acutely angulated branching vessels. In our study, we prospectively evaluated the feasibility and safety of selective embolization with coils by using temporary coil protection of the branch arising from the aneurysm neck.

\section{MATERIALS AND METHODS \\ Population}

At our institution, a total of 1417 saccular aneurysms (1263 patients) were treated by endovascular coil embolization between August 2007 and December 2011. Therapeutic alternatives were discussed between neurosurgical and neurointerventional teams in a multidisciplinary decision-making process. Our method of coil protection was applied to 12 aneurysms ( 8 female and 4 male patients; mean age, $60.5 \pm 9.9$ years). Only 2 patients presented with subarachnoid hemorrhage, indicative of rupture, and their clinical status was Hunt and Hess scale grade II. Eleven aneurysms 

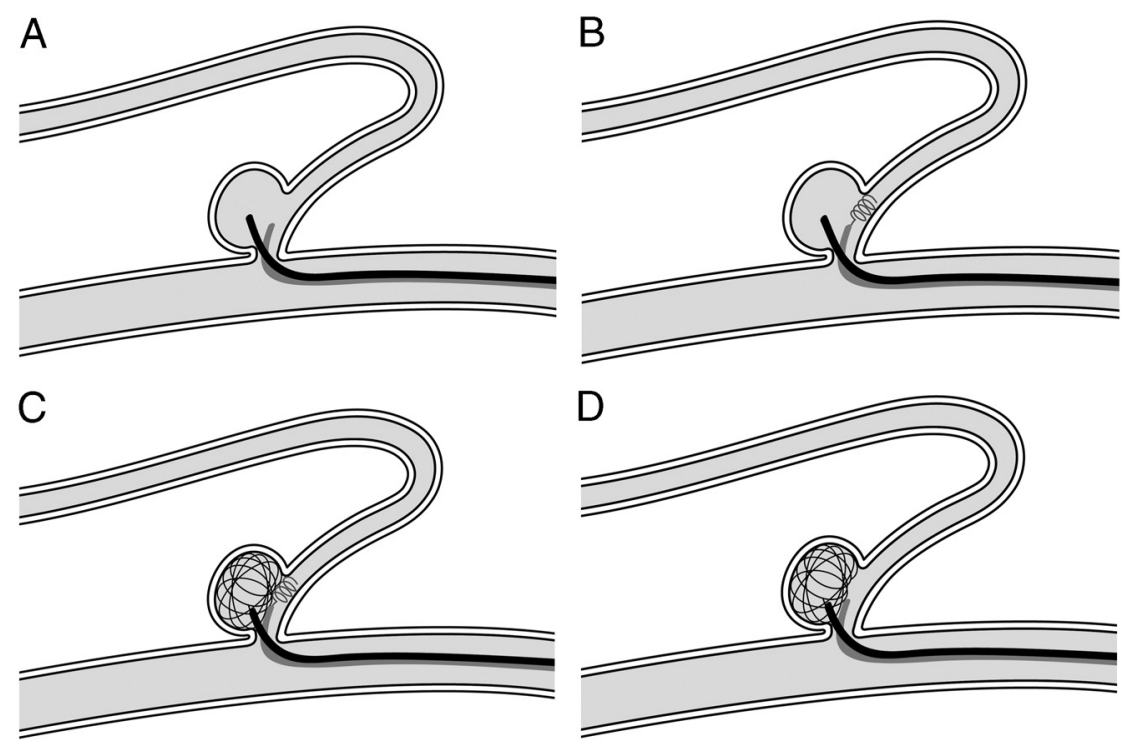

FIG 1. A, Microcatheter for coil delivery to the aneurysmal sac; second microcatheter delivering the protection coil at the orifice of the branch artery. $B$, Small helical protection coil deployed at the lumen of the branch artery near the aneurysm neck. $C$, With coil protection, complex coil insertion into aneurysmal sac. $D$, Confirmation of coil stability by retrieval of the protection coil.

had wide necks, with dome-to-neck ratios $<1.5$. Eleven were small aneurysms, with the longest diameter $<10 \mathrm{~mm}$.

\section{Therapeutic Strategy}

Our technique involved the following steps: 1) microcatheter delivery of the coil for filling the aneurysm, 2) microcatheter (second) delivery of the coil for protecting the branch artery, 3) partial deployment of a small helical coil (via the second microcatheter) into the branch artery near the aneurysmal neck for protection, 4) framing the aneurysm with the first coil under protection of the deployed helical coil, 5) retrieval of the helical coil to confirm the stability of the inserted framing coil, and 6) filling the residual aneurysm with additional coils, including the protection coil (Fig 1).

This technique is not advocated as a first option. If coiling is feasible with other remodeling/protective techniques, coil protection is unnecessary. Coil protection was our resort under the following circumstances: 1) the protection microcatheter or stent/ balloon delivery was prohibited due to an acute angle or small size of the branch artery, 2) the framing coil could not be placed by using multiple microcatheters due to the wide neck of the aneurysm, and 3) flow inhibition or arrest by stent deployment was expected due to the acute angle or small size of the branch artery. Once the first coil was satisfactorily configured under coil protection, we selected 1 of 3 options on the basis of coil stability: 1) continuous insertion of the filling coil under coil protection; 2) insertion of the filling coil via dual microcatheters without coil protection, by using a previously placed microcatheter for protection or another microcatheter with good support; and 3) insertion of the filling coil via double microcatheters under coil protection, by using an additional microcatheter. We confirmed the stability of the frame or filling coil by retrieving the protection coil. The protection coil can be used to fill the remainder of the aneurysmal sac.

\section{Endovascular Procedure}

All procedures were performed with the patient under general anesthesia. Aneurysmal configuration and arterial architecture were evaluated by using the Integris V (Philips Healthcare, Best, the Netherlands) biplane system, including $3 \mathrm{D}$ rotational angiography. Before the procedure, patients with unruptured aneurysms were given single or dual antiplatelet medication, depending on clopidogrel resistance, via a VerifyNow P2Y12 assay (Accumetrics, San Diego, California). Heparin (3000 IU) was administered as a bolus after femoral artery sheath placement; and intermittently thereafter, a 1000-IU bolus per hour was administered with monitoring of the activated clotting time. When rupture was evident, heparinization was deferred until adequate protection of the aneurysms was achieved. An oral antiplatelet agent was routinely administered after the procedure for 1 month or longer, depending on the underlying atherosclerotic compromise or stent placement.

\section{Immediate and Final Outcome}

Immediate angiographic results after coil embolization were assessed by 2 experienced neurointerventionists (M.H.H. and H.S.K.) to document aneurysm obliteration and were categorized into 3 groups: total occlusion (no residual filling of the contrast medium in the aneurysms), near-total occlusion (a small amount of residual contrast filling at the base of the aneurysm), and subtotal occlusion (any contrast filling in the aneurysm sac).

In patients with unruptured aneurysms, MRA with $3 \mathrm{D}$ reconstruction and/or plain radiography was recommended 6, 12, 24, and 36 months after coil embolization. Additional plain radiography was recommended 1 and 3 months postembolization in patients presenting with hemorrhage. Conventional angiography was recommended when assessing the status of the treated aneurysms with MRA was not feasible or when aneurysmal recanalization was suspected by a noninvasive evaluation, such as MRA or plain radiography, to decide whether further treatment was necessary.

Follow-up results were categorized as follows: stable occlusion (no filling of the aneurysm on MRA or angiography and no change of the coil configuration on plain radiography), minor recanalization (slight filling at the neck of the aneurysm on MRA or angiography and minimal coil compaction on plain radiography), and major recanalization (flow filling of the aneurysmal sac and prominent coil compaction).

\section{RESULTS}

Selective endovascular treatment was successfully performed and resulted in excellent outcomes in all patients. Twelve patients with saccular aneurysms of the MCA bifurcation (5 patients); anterior communicating artery (3 patients); and A1 and M1 segments, 

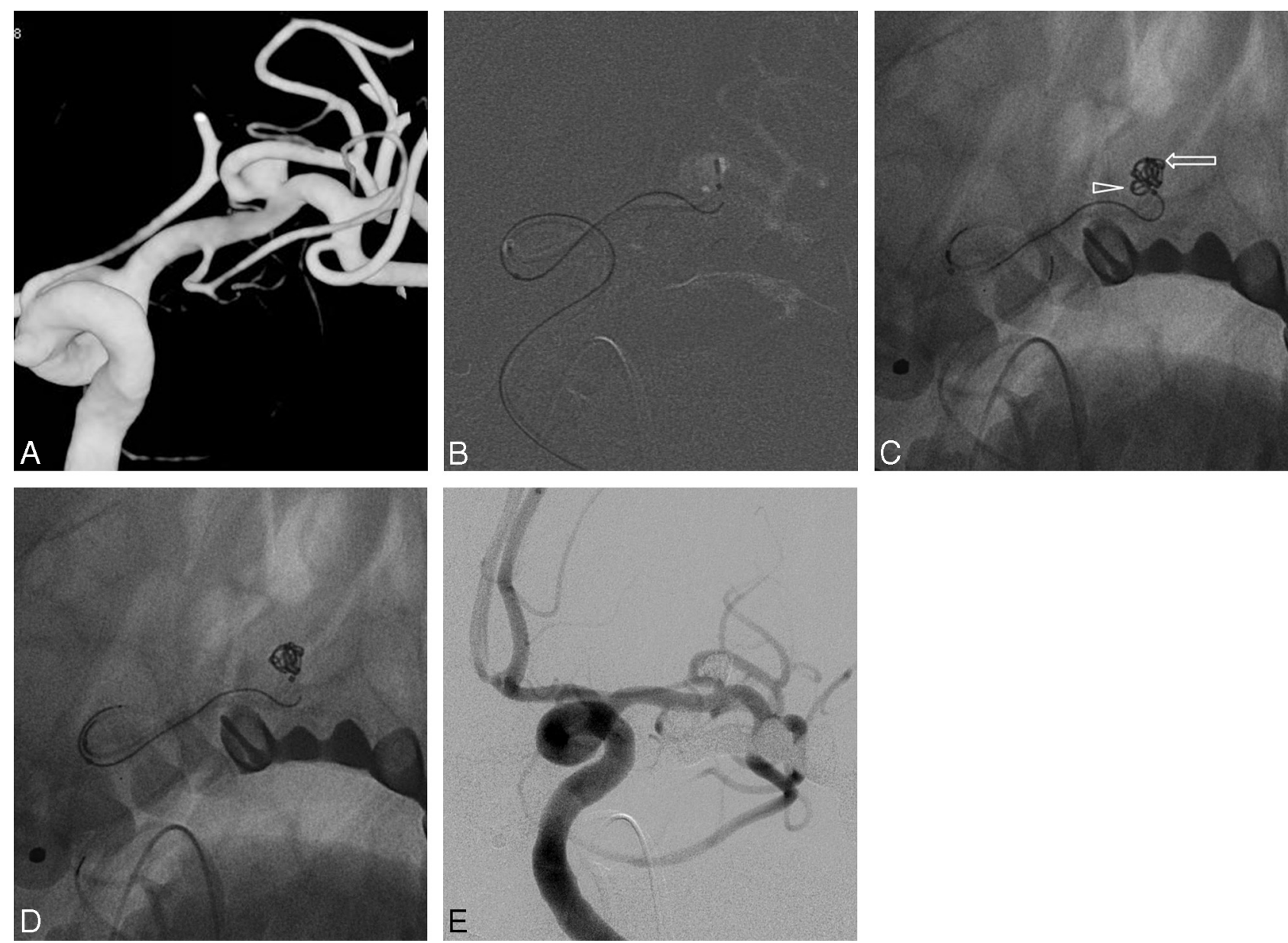

FIG 2. $A, 3 D$ reconstruction image of a wide-neck $\mathrm{Ml}$ aneurysm. $B$, Microcatheter at the entrance of the branch artery; a second microcatheter in the aneurysmal sac. $C$, Small helical coil $(2 \times 4 \mathrm{~cm})$ deployed partially at the lumen of the branch artery near the distal neck of the aneurysm; complex coil inserted under protection (complex coil within the aneurysmal sac at the arrow; arrowhead indicating the helical protection coil). $D$, Coil stability is confirmed by retrieving the protection coil. E. Additional coils inserted under protection plus a helical coil (used for protection); near-total occlusion of aneurysm.

distal ACA, and basilar tip (1 patient each) were successfully treated by our technique. All except 1 patient did not experience any procedural complication and did not have any neurologic sequelae. Although thrombotic occlusion due to coil protrusion (not to coil protection) occurred in 1 instance, it was resolved with intra-arterial tirofiban infusion and stent deployment, and the patient recovered fully. In the immediate aftermath of coil embolization, 10 aneurysms displayed near-total occlusion and 2 showed subtotal occlusion, with a packing attenuation of $27 \%$ and 28\%, respectively. Eight patients (4 treated recently were exempt) underwent follow-up evaluations, including MRA and conventional angiography. Five maintained stable coil configurations, while the others showed recanalization. Major recanalization was demonstrated in 1 patient who underwent additional embolization with coils. None of the patients experienced delayed complications such as thromboembolic infarction or hemorrhage.

\section{Illustrative Case 1}

This 63-year-old woman was admitted for endovascular treatment of an unruptured M1 segment aneurysm found on MR angiography. By conventional angiography, a wide aneurysmal neck was noted, and the M1 branch arising from the parent artery was small caliber with an acute angle. A $6 \mathrm{~F}$ guiding catheter was placed in the cervical segment of the left internal carotid artery, with initial plans to apply microcatheter protection. After placement of a microcatheter in the aneurysmal sac, another microcatheter was inserted into the anterior frontal artery, but it could not be passed due to the steep slope of the small artery and the lack of support. The first coil inserted protruded into the lumen of the branch artery, so we positioned the microcatheter tip near the aneurysmal neck and partially deployed the helical coil. Under helical coil protection, the frame of the first coil was then configured satisfactorily. We confirmed stability of the first coil by retrieving the protection coil, and an additional coil was inserted to fill the entire sac. The helical coil was also inserted as a filler. Near-total occlusion of the aneurysm was achieved (Fig 2), and the patient was discharged the next day without complications.

\section{Illustrative Case 2}

This 60-year-old woman with an MCA bifurcation aneurysm presented with dizziness. The aneurysm had a wide neck and a shallow configuration. Despite a double-microcatheter technique applied to form the frame with a complex coil at first, the coil still 

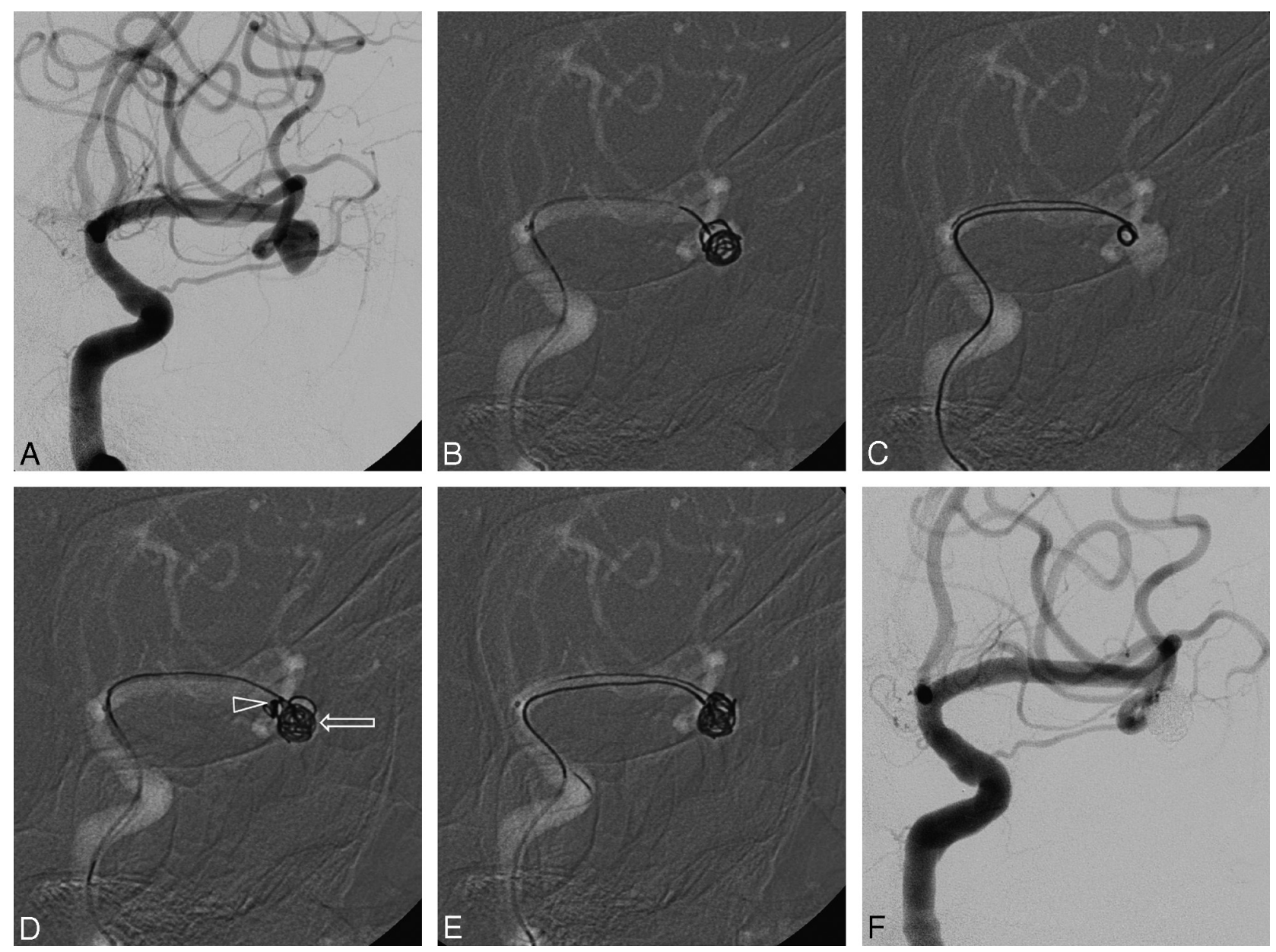

FIG 3. $A$, MCA bifurcation aneurysm seen with conventional angiography. $B$, Coil protruding into the parent artery (an attempt to form a frame with the complex coil by using dual microcatheters). C, Helical protection coil deployed at the entry of the inferior branch of the left MCA. D, Frame coil inserted under protection (a frame coil within the aneurysmal sac at the arrow; arrowhead indicating helical protection coil). E, Microcatheter for protection inserted into the aneurysmal sac. F, Protection coil inserted into the sac via dual microcatheters.

protruded into the parent artery through the inferior portion of the aneurysmal neck. A microcatheter-delivering protection coil was then placed at the entry to the inferior branch of the left MCA. Under coil protection, a complex coil was inserted and formed a good frame without protrusion. The protection microcatheter was later inserted into the aneurysmal sac, and additional coils were used to fill the remainder of the aneurysm by using dual microcatheters. The aneurysm was satisfactorily occluded without complications (Fig 3).

\section{DISCUSSION}

This preliminary study with a short series suggests that saccular intracranial wide-neck aneurysms arising from small and acutely angulated branching vessels may be safely and efficiently treated by this new coil protection endovascular approach. Anatomic results and clinical outcomes are encouraging, especially because the selected aneurysms were judged difficult using the regular endovascular treatment.

Although coil embolization of aneurysms with wide necks is challenging, available remodeling or protective techniques have significantly widened the applicability of endovascular therapy for wide-neck aneurysms. ${ }^{1-7}$ Nevertheless, these methods are not sufficient to treat a few aneurysms, depending on aneurysmal configurations and the course or caliber of the branch artery. In instances in which the branch artery is small and departs from the parent vessel at an acute angle, these techniques are potentially dangerous, entailing such hazards as aneurysmal irritation, vasospasm, flow arrest, and arterial dissection. Coil protection can be helpful in this setting by facilitating an initial frame for complex coils. With coil protection, the frame coil is prevented from protruding into the branch lumen at the aneurysmal neck. The shape of the microcatheter is very important to properly position the protective coil. It is best when the microcatheter tip veers naturally into the branch orifice, which, in some cases, necessitates a $C$ or $S$ preshape.

A small-sized helical coil maintains a rounded shape to concentrate at 1 point, affording better protection than large-sized or complex coils. This characteristic of helical coils could prevent the protective coil from moving distally by flow during deployment and could augment the protective effect of the coil. There also seem to be fewer thromboembolic complications attributable to small helical bare coils because these might maintain the overall flow through the small helical loops. The helical coil should be 
stretch-resistant due to the necessity of repetitive movements back and forth.

The isolated thrombotic event encountered with one of our patients was related to coil protrusion during the process of final insertion. Coil protection was only invoked to form the initial frame. We later reverted to multiple-microcatheter use (as with the patient in illustrative case 2, Fig 3). Although the above was not directly related to coil protection, some thromboembolic risk may still be conferred. Antiplatelet preparations and anticoagulant infusion are mandatory to reduce this potential. Restrictive use of coil protection is recommended in patients with ruptured aneurysms due to the limitation of the antithrombotic strategies, though there were 2 such patients in our series. Physicians should also restrict the overall time for deploying the protection coil to a minimum. Despite the fact that no symptomatic thromboembolic events were documented during the procedure, no advanced noninvasive neuroimaging (such as DWI) was performed immediately after the procedure to ascertain that no potential asymptomatic embolic events occurred in the involved vascular territory. This is one of the study limitations. Interventionists should also be aware of the possibility of coil entanglement between the protection coil and the framing coil, though such a hazardous event did not occur in our series.

We do not advocate coil protection as a first-line technique for embolization of wide-neck aneurysms. The efficacy and safety of this approach must be established with a larger study population. However, it may constitute a viable alternative in disadvantaged situations in which standard methods do not apply.

\section{CONCLUSIONS}

Having successfully treated 12 aneurysms with wide necks by using the coil-protection technique, we believe it seems to be a safe and feasible alternative to traditional remodeling or protective techniques when these have failed or are not possible due to vascular geometry. Coil protection is particularly suited for the treatment of wide-neck aneurysms arising from small and acutely an- gulated branching vessels. However, a larger series with long-term follow-up is mandatory to confirm these preliminary results in terms of safety and efficacy.

\section{ACKNOWLEDGMENTS}

We thank Yoon-Kyung Choi for preparation of the illustrations.

Disclosures: Moon Hee Han-UNRELATED: Consultancy: MicroVention. * *Money paid to the institution.

\section{REFERENCES}

1. Gordhan A. Microcatheter neck bridging and incorporated branch vessel protection for coil embolization of a wide-neck ruptured aneurysm: technical case report. Neurosurgery 2011;68:40-43

2. Peluso JP, van Rooij WJ, Sluzewski M, et al. A new self-expandable nitinol stent for the treatment of wide-neck aneurysms: initial clinical experience. AJNR Am J Neuroradiol 2008;29:1405-08

3. Weber W, Bendszus M, Kis B, et al. A new self-expanding nitinol stent (Enterprise) for the treatment of wide-necked intracranial aneurysms: initial clinical and angiographic results in 31 aneurysms. Neuroradiology 2007;49:555-61

4. Shapiro M, Babb J, Becske T, et al. Safety and efficacy of adjunctive balloon remodeling during endovascular treatment of intracranial aneurysms: a literature review. AJNR Am J Neuroradiol 2008; 29:1777-81

5. Pierot L, Cognard C, Spelle L, et al. Safety and efficacy of balloon remodeling technique during endovascular treatment of intracranial aneurysms: critical review of the literature. AJNR Am J Neuroradiol 2012;33:12-15

6. Lee JY, Seo JH, Cho YD, et al. Endovascular treatment of wide-neck intracranial aneurysms using a microcatheter protective technique: results and outcomes in 75 aneurysms. AJNR Am J Neuroradiol 2011;32:917-22

7. Biondi A, Janardhan V, Katz JM, et al. Neuroform stent-assisted coil embolization of wide-neck intracranial aneurysms: strategies in stent deployment and midterm follow-up. Neurosurgery 2007; 61:460-68

8. Lubicz B, Lefranc F, Levivier M, et al. Endovascular treatment of intracranial aneurysms with a branch arising from the sac. AJNR Am J Neuroradiol 2006;27:142-47 